\title{
Left ventricle pseudoaneurysm as late postoperative complication of a large apical aneurysm
}

\author{
Mariana M. Floria ${ }^{1,4}$, Carmen Elena Pleșoianu ${ }^{2,4}$, Michel Buche ${ }^{3}$, Baudouin Marchandise ${ }^{4}$, Erwin \\ Schroeder ${ }^{5}$ \\ 1. III Medical Clinic, "Sf. Spiridon" University Hospital and "Grigore T. Popa” Medicine and Pharmacy University, Iasi, \\ Romania. 2. Cardiovascular Disease Institute, "Grigore T. Popa" Medicine and Pharmacy University, Iasi, Romania. 3. \\ Cardiovascular Surgery Unit, CHU Mont-Godinne,Universitè catholique de Louvain, Yvoir, Belgium. 4. Echocardiography \\ Unit, CHU Mont-Godinne, Universitè catholique de Louvain, Yvoir, Belgium. 5. Cardiology Unit, CHU Mont-Godinne, \\ Universitè catholique de Louvain, Yvoir, Belgium.
}

Correspondence: Mariana M. Floria. Address: Bd. Dacia, nr. 1, bl DA3, et 10, ap 40, 700425, Iasi, Romania. Email: floria_mariana@yahoo.com

Received: February 12, 2014

Accepted: March 2, 2014

Online Published: March 20, 2014

DOI: $10.5430 /$ ijdi.v1n2p33

URL: http://dx.doi.org/10.5430/ijdi.v1n2p33

\begin{abstract}
We report here the case of a patient with large left ventricular apical aneurysm incidentally discovered seven years after an inferior myocardial infarction. The aneurysm was successfully corrected by surgery. However, five months after surgery, the patient's evolution was complicated by the sudden occurrence of a pseudoaneurysm. This case is complex and interesting because of several factors, including the incidental discovery, the atypical localization of an aneurysm secondary to the right coronary chronic occlusion, the size of the aneurysm, the preoperative differential diagnosis and the unusual late postoperative evolution.
\end{abstract}

\section{Keywords}

Left ventricular, Myocardial infarction, Aneurysm, Pseudoaneurysm

\section{Introduction}

The incidence of left ventricle (LV) aneurysms is decreasing, thanks to major improvements in the management of patients with acute myocardial infarction, e.g., by primary percutaneous coronary intervention and thrombolytic therapy. Aneurysms of the apex and anterior wall are four times as common as those of the inferior or inferoposterior walls ${ }^{[1,2]}$. Major complications include heart failure, ventricular arrhythmia and thromboembolism. A true LV aneurysm rarely ruptures, because of its densely fibrotic wall, unlike pseudoaneurysm, a rare complication of inferior myocardial infarction, which frequently has a fatal outcome ${ }^{[3]}$. Rupture of the free wall of the left ventricle is a catastrophic complication of acute myocardial infarction. Rarely, free wall rupture is contained by overlying adherent pericardium, producing a pseudoaneurysm of the left ventricle. In this report, a case of a left ventricular pseudoaneurysm due to a previous surgically corrected left ventricular aneurysm is described. 


\section{Case presentation}

We report the incidental discovery of a large apical LV aneurysm in a 69-year-old man admitted in the department of pneumology for bacterial pneumonia, with unexpected and tragic late postoperative evolution. From his medical history, it was noted that the patient had type 2 diabetes mellitus and chronic obstructive pulmonary disease complicated by moderate pulmonary arterial hypertension. Seven years earlier, he had also suffered an inferior myocardial infarction treated by percutaneous transluminal coronary angiography and right coronary artery stent placement (single vessel disease). Two-dimensional transthoracic echocardiography revealed a large apical LV aneurysm measuring $78 \mathrm{~mm} \times$ $79 \mathrm{~mm} \times 57 \mathrm{~mm}$ in diameter (see Figure 1) associated with moderate LV dilatation, moderate mitral regurgitation and poor LV systolic function (ejection fraction of $25 \%$ ).

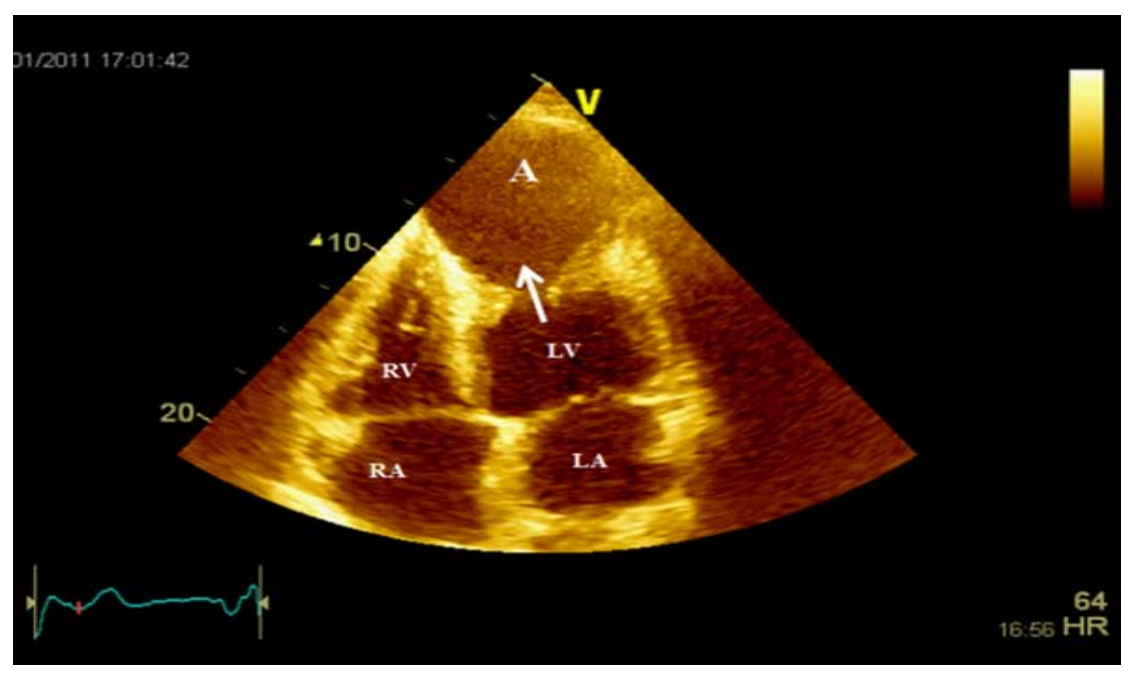

Figure 1. Two-dimensional transthoracic echocardiogram (apical four-chamber view) showing a large cavity "attached" to the $\mathrm{LV}$ chamber. $\mathrm{A}$ = aneurysm, $\mathrm{LA}=$ left atrium, $\mathrm{RA}=$ right atrium, $\mathrm{RV}=$ right ventricle

Coronary angiography revealed stent permeability, right coronary artery dominance irrigating the apex (see Figure 2).
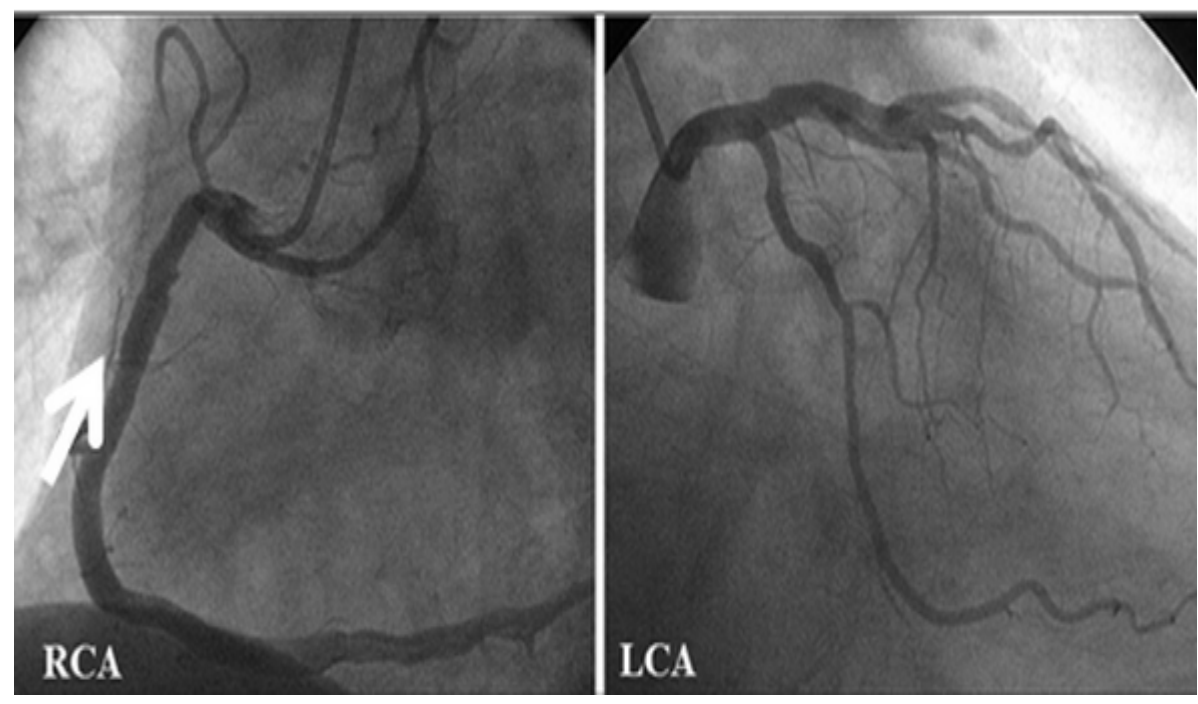

Figure 2. Right (RCA) and left coronary artery (LCA) angiograms showed right coronary artery stent permeability with no new other significant lesions. 
Differential diagnosis between aneurysm and pseudoaneurysm was made by three-dimensional echocardiography (see Figure 3) and contrast ventriculography (see Figure 4), and later confirmed intraoperatively and by anatomopathological assessment.

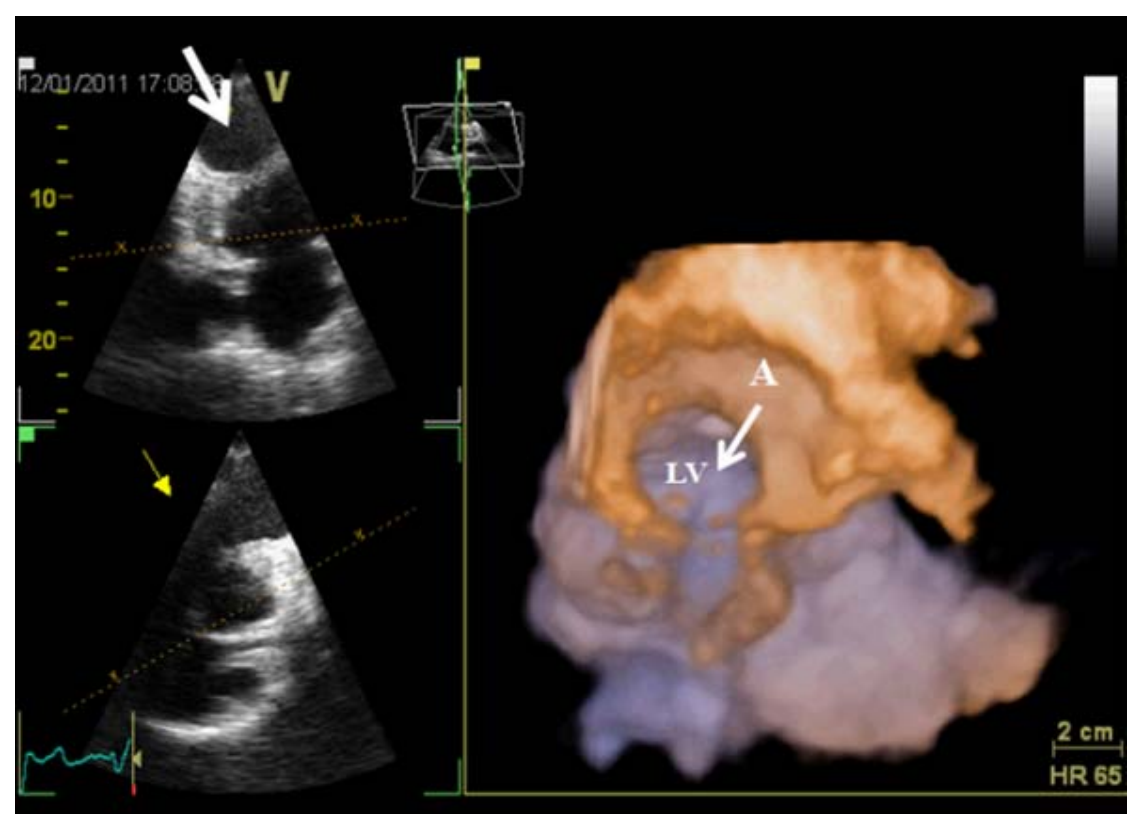

Figure 3. Three-dimensional transthoracic echocardiogram confirming a large communication between the left ventricle (LV) chamber and aneurysm (A).

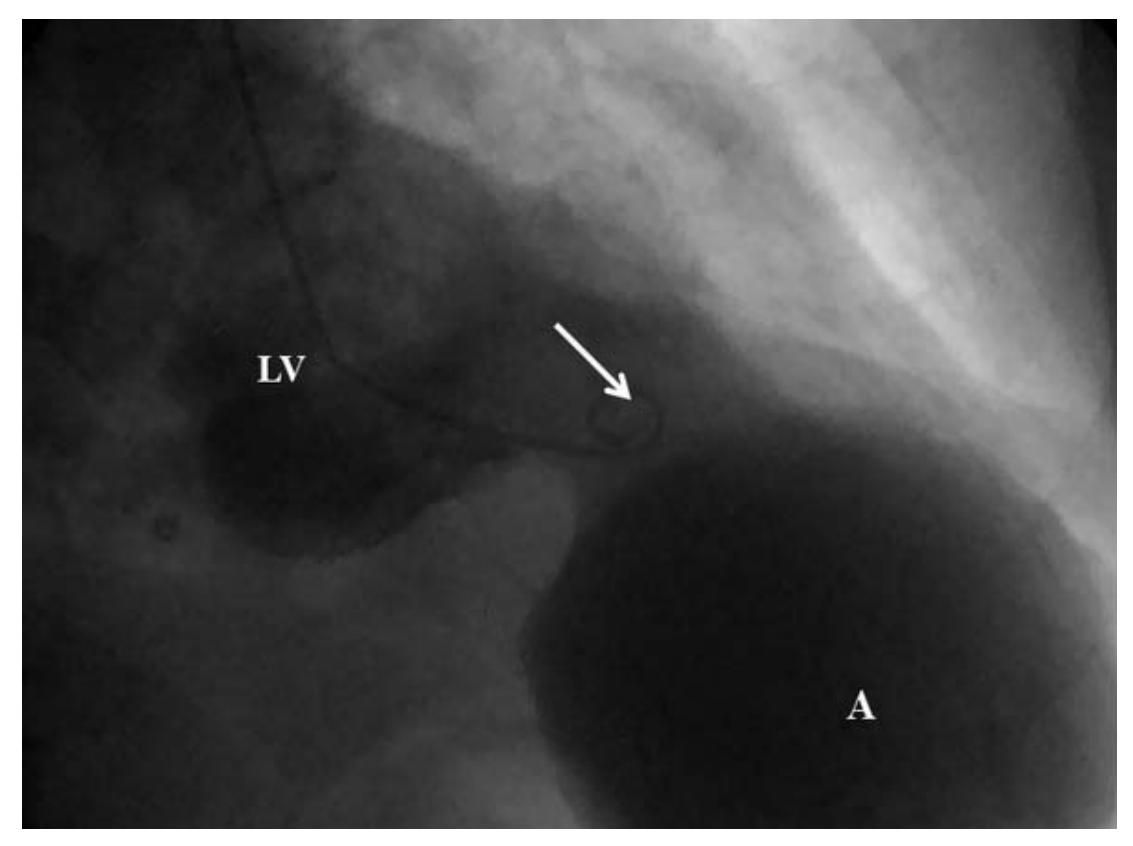

Figure 4. Contrast ventriculography showing a large left ventricle (LV) aneurysm (A).

LV aneurysmectomy was performed by reconstruction with a bovine pericardial patch reinforced by the thin autologous aneurysmal wall (see Figure 5). 


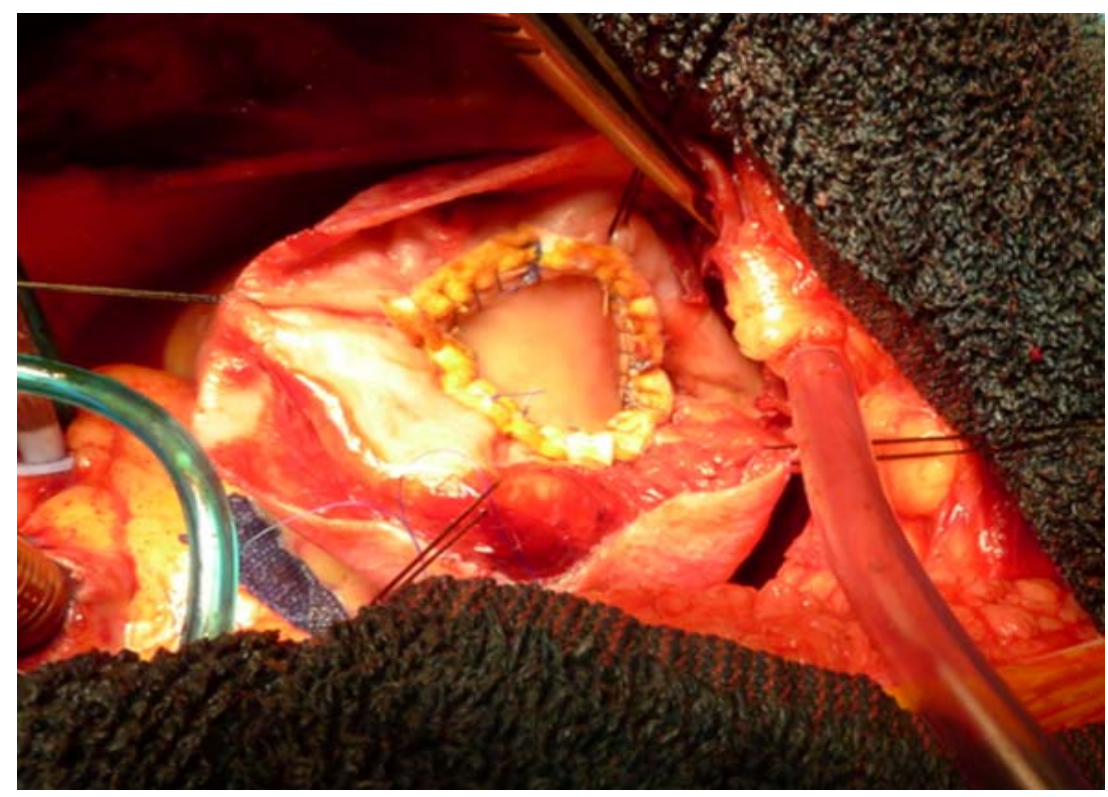

Figure 5. Perioperative image of the aneurysm and LV apex reconstruction

During hospitalization, LV systolic function significantly improved under classical medical management of heart failure. Clinical and echocardiographic evolution was excellent. At four months, the LV ejection fraction reached 50\%, confirmed by transthoracic echocardiography and magnetic resonance imaging. Unfortunately, one month later, the patient was admitted to the emergency department with chest pain and acute dyspnea, associated with elevated cardiac enzymes and ST segment elevation in the inferiolateral leads. Two-dimensional transthoracic echocardiography, corroborated by magnetic resonance imaging, revealed a normal LV cavity with a slightly decreased LV systolic function and apical and infero-septo-apical akinesis with paradoxical motion of the reconstructed apex during the cardiac cycle. Moreover, a small color Doppler flow was detected between the left ventricular cavity and the aneurysmal wall used for the reconstruction of the apex, which was separated from the pericardial patch, creating an aspect of pseudoaneurysm. A thrombus was also identified in this cavity (see Figure 6).

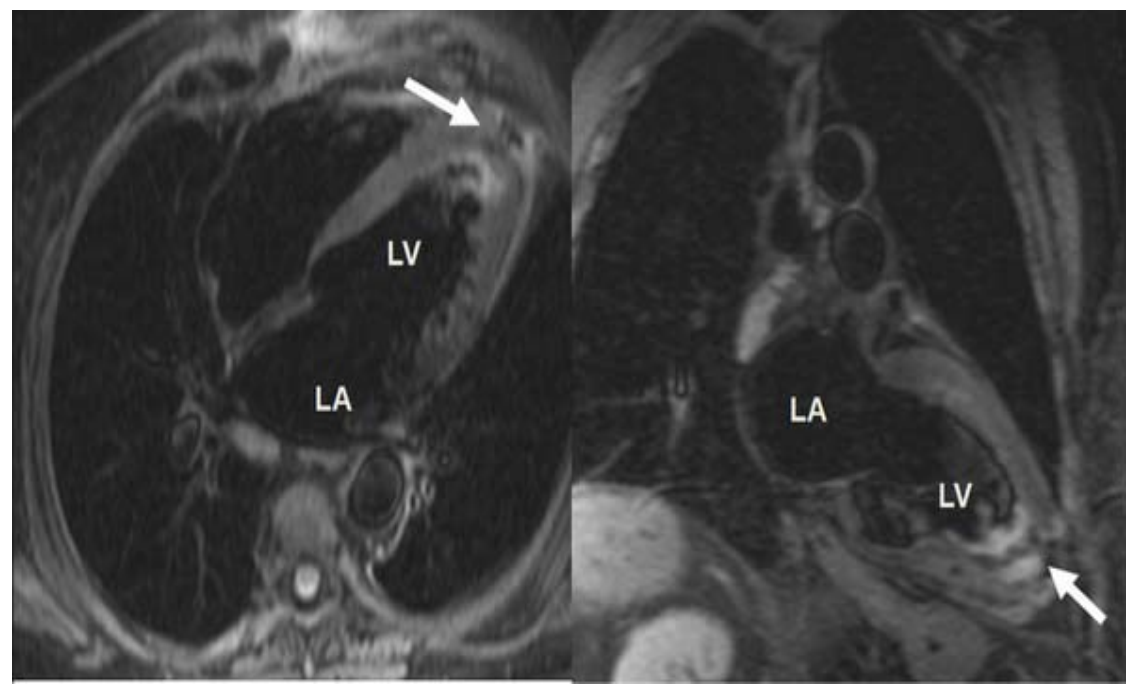

Figure 6. Magnetic resonance imaging performed upon admission at five months and showing an aspect of apical pseudoaneurysm with thrombus inside (in four and two apical views). LA = left atrium; LV = left ventricle. 
The patient died soon after admission from asystole, unresponsive to cardiopulmonary resuscitation, before surgical reintervention.

\section{Discussion}

Pseudoaneurysm originating from left ventricle aneurysm is very rare ${ }^{[4]}$. It can appear after sutureless repair of left ventricular free wall rupture due to acute myocardial infarction ${ }^{[5]}$ or post thoracic trauma ${ }^{[6]}$. Sometimes the pseudoaneurysm can be first diagnosed at autopsy ${ }^{[4]}$.

In the present case, the large apical aneurysm with poor LV systolic function was incidentally diagnosed, probably mainly because the dyspnea was considered to be due to the chronic obstructive pulmonary disease. Two- and three-dimensional transthoracic echocardiography and resonance magnetic imaging have a major role in the diagnosis of aneurysms, detection of related complications and postoperative follow-up ${ }^{[1]}$. Closer follow-up is mandatory in these patients. The optimal management of a patient with a large, asymptomatic LV aneurysm remains a clinical dilemma. Surgical repair of the aneurysm is recommended when coronary artery bypass surgery or valve surgery are indicated, but also when a progressive increase in LV diameter and/or decrease in LV ejection fraction are noted ${ }^{[2,3]}$. The procedure is very effective and results in a significant improvement in patient survival, symptoms and functional class, compared to medical treatment ${ }^{[2,3]}$. Here, there was an appropriate indication for surgery, although multiple independent predictors of increased early and long-term postoperative mortality were identified, including the patient's age, poor LV function and moderate mitral regurgitation ${ }^{[7]}$. However, after surgery, postoperative cardiac index and LV ejection fraction were markedly improved. The unfavorable evolution, complicated by the occurrence of a pseudoaneurysm, could be explained in the context of possible undiscovered hypertension with a partial detachment of the pericardial patch. A small color Doppler flow was detected between the left ventricular cavity and the aneurysmal wall used for the reconstruction of the apex, which was separated from the pericardial patch. It rapidly led to a secondary rupture with fatal outcome before the possibility of reintervention. An additional infarction of the apex could be also incriminated. The patient died soon after readmission. Unfortunately anatomopathological examination was not possible (because of a staff problem).

\section{Conclusion}

Left ventricular pseudoaneurysm as a late complication of a previous surgically corrected left ventricular aneurysm is very rare. Closer follow-up is mandatory in these patients. This case is complex and interesting because of several factors, including the atypical localization of a left ventricular aneurysm secondary to the right coronary chronic occlusion, the size of the aneurysm, the incidental discovery, the preoperative differential diagnosis and the unusual postoperative evolution.

\section{References}

[1] Heatlie GJ, Mohiaddin R. Left ventricular aneurysm: comprehensive assessment of morphology, structure and thrombus using cardiovascular magnetic resonance. Clin Radiol. 2005; 60: 687. http://dx.doi.org/10.1016/j.crad.2005.01.007

[2] Antman EM, Anbe DT, Armstrong PW, et al. ACC/AHA guidelines for the management of patients with ST-elevation myocardial infarction. J Am Coll Cardiol. 2004; 44(3): E1-E211. http://dx.doi.org/10.1016/j.jacc.2004.07.014

[3] Antman EM, Hand M, Armstrong PW, et al. 2007 focused update of the ACC/AHA 2004 Guidelines for the Management of Patients With ST-Elevation Myocardial Infarction: a report of the American College of Cardiology/American Heart Association Task Force on Practice Guidelines (Writing Group to Review New Evidence and Update the ACC/AHA 2004 Guidelines for the Management of Patients With ST-Elevation Myocardial Infarction). J Am Coll Cardiol. 2008; 51(2): 210-47. http://dx.doi.org/10.1016/j.jacc.2007.10.001

[4] Dogan KH, Demirci S, Tavli L, Buken B. Pseudoaneurysm originating from left ventricle aneurysm: an autopsy case and review of literature. J Forensic Leg Med. 2013; 20(8): 941-3. http://dx.doi.org/10.1016/j.jflm.2013.09.004 
[5] Hamamoto M1, Morifuji K. Surgery for left ventricular pseudoaneurysm: thoracotomy or sternotomy. Asian Cardiovasc Thorac Ann. 2013; 21(5): 602-4. http://dx.doi.org/10.1177/0218492312459858

[6] Chen F, Wei S, Xiong L, Liu F. Post-traumatic left ventricular outflow tract pseudoaneurysm. Ann Thorac Surg. 2014; 97(1): 311-2. http://dx.doi.org/10.1016/j.athoracsur.2013.04.137

[7] Lundblad R, Abdelnoor M, Svennevig JL. Surgery for left ventricular aneurysm: early and late survival after simple linear repair and endoventricular patch plasty. J Thorac Cardiovasc Surg. 2004; 128: 449. http://dx.doi.org/10.1016/j.jtcvs.2004.04.017 